\title{
Beteiligung der Lunge beachten, alternative Wege einschlagen und Komplikationen vermeiden
}

\section{Blickdiagnosen \\ Welche Diagnose vermuten Sie? FOKUS}

Ein 65-jähriger Mann wurde mit Übelkeit, Erbrechen und Dehydrierung stationär aufgenommen. 6 Monate zuvor war bei ihm ein Adenokarzinom der Prostata (Gleason-Grad $3+4$ ) diagnostiziert worden, und 6 Wochen vor der Aufnahme hatte er eine Strahlenbehandlung des Beckens abgeschlossen. Außerdem lag in der Anamnese eine rheumatoide Arthritis vor, die mit Methotrexat behandelt worden war, bis das Arzneimittel 2 Wochen vor der Aufnahme wegen Panzytopenie und erhöhter Leberenzymwerte abgesetzt worden war.

Eine körperliche Untersuchung war ohne Befund; die gemessenen Vitalzeichen waren normal. Laboranalytisch lagen bei der Aufnahme ein Hämoglobinwert von 11,2 g/dl und eine Thrombozytenzahl von 90.000 vor. Die Serumwerte für Elektrolyte, Glukose, Blut-Harnstoff-Stickstoff und Kreatinin waren normal. Elektrokardiogramm und Röntgen-Thorax waren unauffällig.

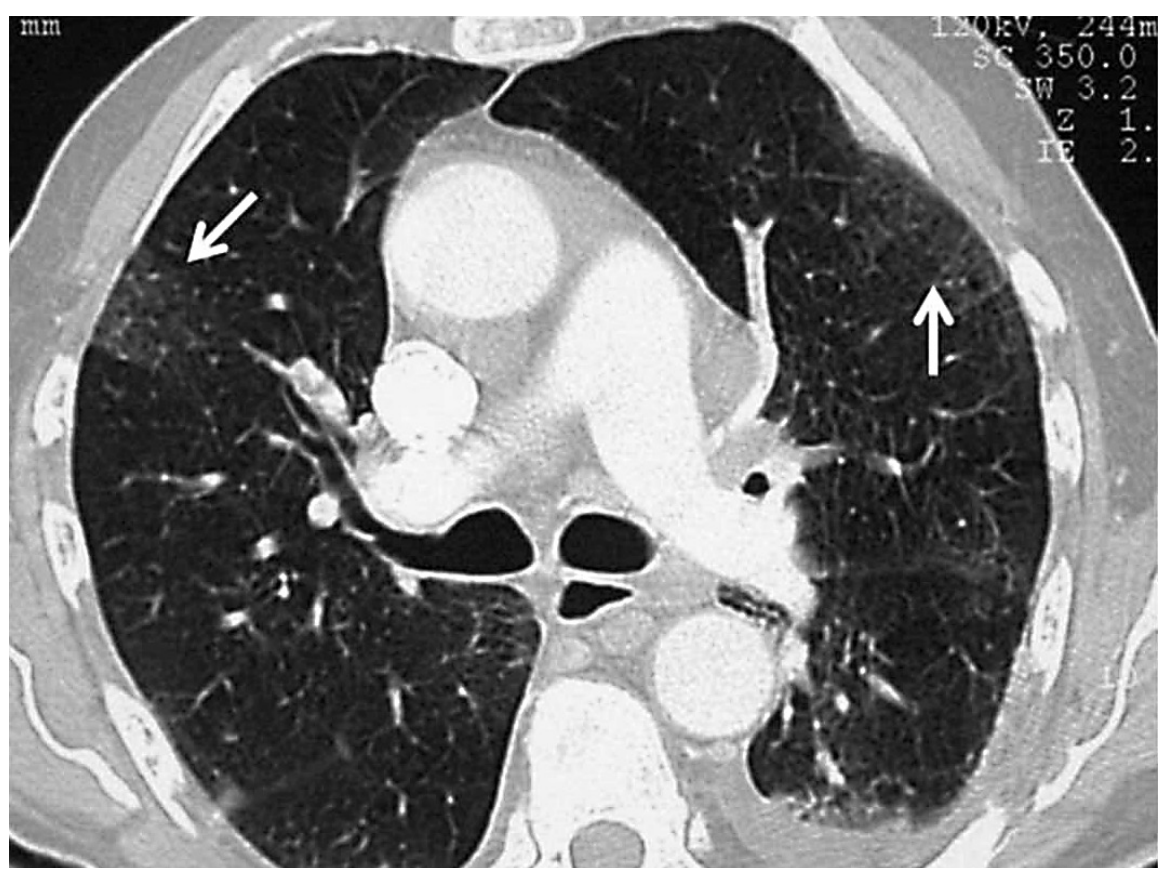

Das CT-Angiogramm des Thorax zeigt einige verstreute Areale schwacher peripherer Milchglastrübungen (Pfeile) ohne Anzeichen einer Lungenembolie. Linksseitig ist auch ein kleiner Pleuraerguss zu sehen.
Während der Aufnahme fielen der Hämatokrit und die Thrombozytenzahl weiter ab, und im peripheren Blutausstrich wurden mit einem erhöhten Serum-LDH-Wert von 12.000 U/I Anzeichen von Hämolyse festgestellt. Die Verdachtsdiagnose einer thrombotischen thrombozytopenischen Purpura wurde gestellt und eine Plasmapherese eingeleitet. Nach 2 Tagen Plasmapherese hatte sich die Thrombozytenzahl stabilisiert; jedoch begann der Patient über Dyspnoe zu klagen, und es wurde eine Sauerstoffsättigung von $85 \%$ in Raumluft gemessen. In einer CT-Angiographie des Thorax (s. Abb.) zeigten sich keine Anzeichen einer Lungenembolie. Im Echokardiogramm zeigte sich eine Vergrößerung und eine verminderte systolische Funktion des rechten Ventrikels bei einem geschätzten rechtsventrikulären systolischen Druck von 74 $\mathrm{mmHg}$. Ein 2 Monate zuvor erstelltes Echokardiogramm des Patienten war ohne Befund. Die Hypoxämie verschlechterte sich, und der Patient wurde auf die Intensivstation verlegt. Es wurde ein Pulmonaliskatheter eingeführt, der einen Pulmonalarteriendruck von 64/24 $\mathrm{mmHg}$, einen Pulmonalkapillar-WedgeDruck von $8 \mathrm{mmHg}$ und einen Herzindex von $3,7 \mathrm{l} / \mathrm{min} / \mathrm{m}^{2}$ ergab. Wie lautet Ihre Diagnose?

Übersetzung aus Respiration. 2014;87: 249-251, DOI: 10.1159/000355321

\section{Auflösung unter: www.karger.com/blickdiagnose- kkp-3-2020}

Kontakt: Eric S. Bensadoun, MD, MPH, Division of Pulmonary and Critical Care Medicine, University of Kentucky, 740 South Limestone, L543 Kentucky Clinic, Lexington, KY 40536-0298, USA, E-Mail ebens0@ uky.edu 


\section{Berufsfelder für Mediziner}

\section{Alternative Karrierewege: Arbeiten in der Pharmabranche}

Wenn es um die Arbeit als Mediziner geht, stellt sich für die meisten die Frage: Klinik oder Praxis? Doch daneben gibt es auch noch andere Möglichkeiten: zum Beispiel einen Job in der pharmazeutischen Industrie.

Das Medizinstudium bietet eine große Bandbreite an späteren Beschäftigungsmöglichkeiten außerhalb von Klinik und Praxis. Neben der Forschung an einer Universität, der Arbeit in einer Unternehmensberatung, dem Medizinjournalismus und dem medizinischen Dienst der Krankenkassen ist auch die Arbeit in einem Pharmaunternehmen eine Option.

Hier gibt es viele Jobs für verschiedene Karrierestufen - also sowohl für junge Mediziner, die direkt von der Uni kommen, als auch für Fachärzte und Spezialisten mit Berufserfahrung. In ihrem Vortrag beim Operation Karriere-Kongress in Berlin stellte Dr. Dina DomrösZoungrana, Trainee Medizin bei Berlin-Chemie, verschiedene Tätigkeitsfelder für Ärzte in der Pharmabranche vor.

\section{Manager für wissenschaftliche Kommunikation (Berufseinsteiger)}

Wer die Pharmabranche kennenlernen möchte, findet hier eine sinnvolle Einstiegsposition. Manager für wissenschaftliche Kommunikation beantworten vor allem Fragen von Ärzten, Apothekern und Patienten zu bestimmten Medikamenten. Außerdem gehören zu ihren Aufgaben:

- Schulung von Außendienstlern

- Organisation von Fortbildungsveranstaltungen und Kommunikation mit den Referenten

- Zusammenarbeit mit Fachreferenten

- Mitarbeit in der Local Drug Safety Unit (LDSU) und im Marketing

- Marktanalyse und Literaturrecherche

\section{Traineeprogramm für Mediziner (Berufseinsteiger)}

Das Traineeprogramm bei Berlin-Chemie ist beispielsweise auf 2 Jahre angelegt. In dieser Zeit rotieren die Trainees durch die wichtigsten Abteilungen und haben die Möglichkeit, sich gut in den verschiedenen Bereichen zu vernetzen, z.B.:

- Gesundheitsmanagement

- Arzneimittelsicherheit

- Marktforschung

- Arzneimittelzulassung

Um in das Traineeprogramm aufgenommen zu werden, ist eine Promotion vorteilhaft. Pflicht ist sie aber nicht. Und man sollte sich nicht von dem Begriff «Trainee» abschrecken lassen: Dabei handelt es sich um ein Programm, dessen Anspruch durchaus mit einer Facharztweiterbildung vergleichbar sei, erklärte Domrös-Zoungrana, die selbst ein Traineeprogramm absolviert.

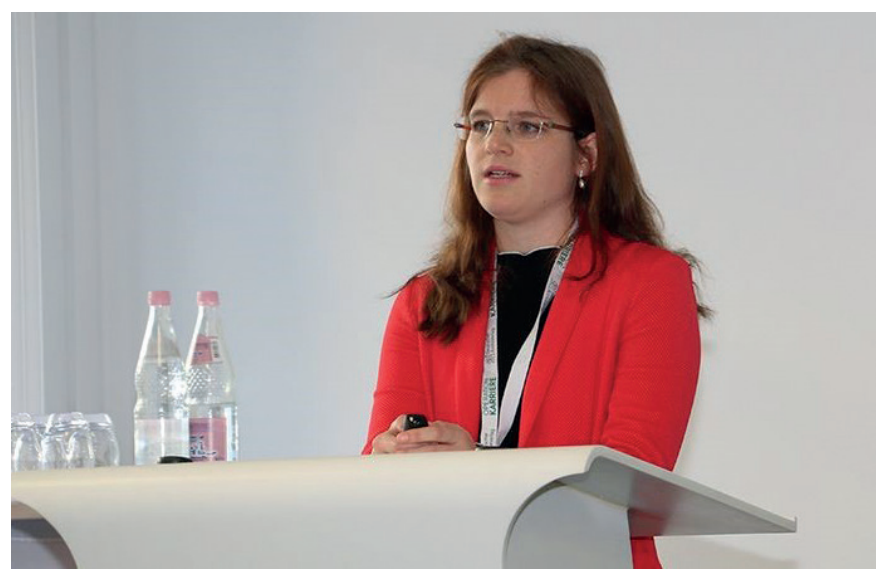

Dr. Dina Domrös-Zoungrana arbeitet als Trainee bei Berlin-Chemie. Beim Operation Karriere-Kongress in Berlin beschrieb sie die Beschäftigungsmöglichkeiten, die es für Mediziner in der Pharma-Branche gibt. @ Hanke

\section{Medical Advisor (Mediziner mit Berufserfahrung)}

Für die Arbeit als Medical Advisor ist Berufserfahrung wichtig: Dieser Job ist sehr anspruchsvoll und kommt vor allem für fertig ausgebildete Fachärzte in Frage. Dabei braucht jedes Medikament einen Medical Advisor, der das Produkt medizinisch-wissenschaftlich betreut. Dazu gehören unter anderem folgende Aufgaben:

- Konzeption, Organisation, Auswertung und Veröffentlichung von Studien

- Planung und Betreuung von Fortbildungsveranstaltungen, Betreuung der Referenten

- Erstellung und Prüfung von Materialien für Ärzte, Patienten, Apotheken und Außendienst

- Durchführung von Schulungen

- Beantworten von medizinischen Fragestellungen für Ärzte, Patienten, Apotheken und Außendienst

Wer erstmal nur in die Pharmabranche hineinschnuppern möchte, kann das übrigens auch gern schon während des Studiums tun: Die Unternehmen bieten unter anderem Jobs für Werkstudenten an, aber auch die Möglichkeit, mal für einen oder mehrere Tage unverbindlich zu hospitieren.

Originalquelle: «Alternative Karrierewege - Ärztliche Tätigkeit in der pharmazeutischen Industrie». Ein Beitrag auf www.operationkarriere.de (www.operation-karriere.de/karriereweg/assistenzarzt/ alternative-karrierewege-arbeiten-in-der-pharma-branche.html)

\section{OPERATION}




\section{Auszeichnungen}

\section{Forschungspreis der Reha Rheinfelden 2019}

Bereits zum 8. Mal hat die Reha Rheinfelden im vergangenen Jahr den Forschungspreis ausgeschrieben. Der Preis ging 2019 an 2 Forscherteams aus dem Bereich Physiotherapie für ihre Studien «Spielerisches, hochintensives Intervalltraining bei älteren Personen» und «Erkennen und Vermeiden des Verschluckens nach einer Lungenoperation» verliehen. Mit dem Preisgeld von 5000 CHF für wissenschaftliche Arbeiten im therapeutischen Bereich wurden beide Teams je zur Hälfte bedacht. Im Mittelpunkt der beiden prämierten Arbeiten standen die folgenden Fragen: Wie lassen sich frühzeitige Immobilität im Alter und Komplikationen nach einer Lungenoperation vermeiden?

Körperliche Inaktivität ist der vierthäufigste Risikofaktor für Sterblichkeit und jährlich für etwa 10\% der Todesfälle verantwortlich. In der ersten de beiden ausgezeichneten Studien befasste sich das aus Stefanie Rebsamen, Ruud H. Knols, Pierrette Baschung Pfister und Eling D. de Bruin bestehende Forschungsteam aus dem Bereich der Physio- und Ergotherapie des Universitätsspitals Zürich, der Zürcher Hochschule für Angewandte Wissenschaften, des Kan-

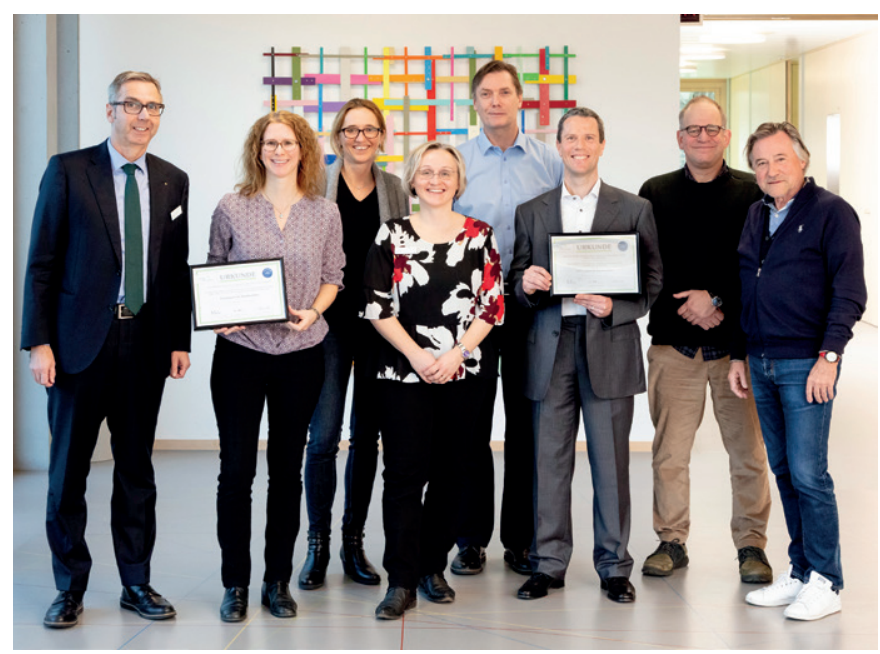

v.l.n.r. Matthias Mühlheim (Adm. Direktor), Stefanie Rebsamen (Forschungsteam) Pierrette Baschung Pfister (Forschungsteam), Dr. Corina Schuster-Amft, PhD (Leiterin wissenschaftliche Abteilung), Prof. Eling de Bruin (Forschungsteam), Joachim M. Schmidt Leuenberger (Forschungsteam), Dr. Ruud H. Knols PhD (Forschungsteam) Prof. Thierry Ettlin (Chefarzt und Med. Direktor). tonsspitals Winterthur und des Instituts für Bewegungswissenschaften der ETH Zürich mit der Durchführbarkeit eines auf virtueller Realität basierten, hochintensiven Intervalltrainings bei 65- bis 80-jährigen Personen. Bei dem 12 Mal über 4 Wochen durchgeführten 20- bis 30-minütigen Training wurden Akzeptanz und Anwendbarkeit des Trainings untersucht. Zusätzlich standen auch Parameter der kardiovaskulären Fitness der älteren Personen im Fokus.

In der zweiten prämierten Arbeit von Joachim M. Schmidt Leuenberger, Beatrix Hoksch, Gere Luder, Ralph A. Schmid, Martin L. Verra und Patrick Dorn untersuchten die Forschenden des Berner Inselspitals den Effekt einer frühzeitigen Behandlung des Verschluckens (Dysphagie) nach einer Lungenresektion. Dabei fokussierten sie sich auf die Vermeidung einer Lungenentzündung und anderer lebensbedrohlicher Komplikationen aufgrund des Verschluckens sowie auf die Länge des Krankenhausaufenthaltes. Innerhalb von 2 Jahren wurden über 430 Patienten behandelt. Im Vergleich zur Kontrollgruppe erkrankten in der behandelten Gruppe signifikant weniger Patienten an einer Lungenentzündung, es gab keine Todesfälle und die Aufenthaltsdauer in der Klinik war kürzer.

Die Jury setzte sich aus den folgenden Mitgliedern zusammen: Prof. Eva Swinnen (Vrije Universiteit Brussel, Belgien), Prof. Erwin Scherfer (Hochschule Furtwangen, Deutschland) und Dr. Corina SchusterAmft (Leiterin wissenschaftliche Abteilung der Reha Rheinfelden, Schweiz).

Die Preisübergabe fand im Rahmen einer großen Fortbildungsveranstaltung der Reha Rheinfelden statt, bei welcher beide Forschungsarbeiten dem interessierten Publikum präsentiert wurden.

Forschungspreis 2020

Der Forschungspreis der Reha Rheinfelden wird jährlich vergeben, so auch in diesem Jahr. Die Bewerbungsunterlagen können noch bis zum 31. August 2020 in deutscher oder englischer Sprache als PDF-Datei per E-Mail an forschungspreis@reha-rhf.ch geschickt werden. Weitere Informationen: Dr. Corina SchusterAmft, Leiterin wissenschaftliche Abteilung, Tel: +41 (0)61 83653 81, c.schuster@reha-rhf.ch, www.reha-rheinfelden.ch/ueber-uns/ wissenschaft/forschungspreis/ 